\title{
SCM-SS scheme for optical MIMO transmission using multimode fibers
}

\author{
Yang Zhang ${ }^{\text {a) }}$, Junya Matsushita, Tomoyasu Nishimori, \\ and Katsushi Iwashita \\ Department of Electronic and Photonic System Engineering \\ Kochi University of Technology \\ Miyanokuchi 185, Tosayamada, Kami, Kochi 782-8502, Japan \\ a)148005f@gs.kochi-tech.ac.jp
}

\begin{abstract}
This letter presents a subcarrier multiplexing with spread spectrum (SCM-SS) scheme for optical multiple-input multiple-output (MIMO) transmission over multimode fibers. Optical MIMO is dedicated to increasing link capacity by exploiting the space domain, which provides a new dimension in addition to conventional time division multiplexing (TDM) and wavelength division multiplexing (WDM). SCMSS scheme is a promising way that avoids resolving channels in optical domain, and multiple channels are recovered by off-line MIMO signal processing. Up to $2000 \mathrm{~m}$ transmissions of $100 \mathrm{Mbps}$ per channel $2 \times 4$ MIMO over graded index multimode fiber link with direct detection have been experimentally demonstrated. Advantageous performance in the presence of intermodal coupling is shown.
\end{abstract}

Keywords: Optical MIMO, LANs, multimode fiber, intermodal coupling, direct detection

Classification: Transmission Systems and Transmission Equipment for Communications

\section{References}

[1] H. R. Stuart, "Dispersive multiplexing in multimode fiber," Science, vol. 289 , no. 5477 , pp. 281-283, July 2000.

[2] A. R. Shah, R. C. J. Hsu, and A. Tarighat, "Coherent Optical MIMO (COMIMO)," J. Lightw. Technol., vol. 23, no. 8, pp. 2410-2419, Aug. 2005.

[3] R. Ryf, S. Randel, and A. H. Gnauck, "Mode-Division Multiplexing Over $96 \mathrm{~km}$ of Few-Mode Fiber Using Coherent $6 \times 6$ MIMO Processing," $J$. Lightw. Technol., vol. 30, no. 4, pp. 521-531, Feb. 2012.

[4] C. P. Tsekrekos, "Design Considerations for a Transparent Mode Group Diversity Multiplexing Link," IEEE Photon. Technol. Lett., vol. 18, no. 22, pp. 2359-2361, Nov. 2006.

[5] B. C. Thomsen, "MIMO enabled $40 \mathrm{~Gb} / \mathrm{s}$ transmission using mode division multiplexing in multimode fiber," Proc. OFC, 2010.

[6] B. Franz, D. Suikat, and R. Dischler, "High Speed OFDM Data Transmission Over $5 \mathrm{~km}$ GI-Multimode Fiber Using Spatial Multiplexing With $2 \times 4$ MIMO Processing," Proc. ECOC, Torino, Italy, pp. 425-427, Sept. 
2010.

[7] A. Tarighat, R. C. J. Hsu, and A. Shah, "Fundamentals and Challenges of Optical Multiple-Input Multiple-Output Multimode Fiber Links," IEEE Commun. Mag., pp. 57-63, May 2007.

\section{Introduction}

Nowadays, the ever growing data traffic in local area networks (LANs) has placed a bottleneck on system performance. Optical MIMO has great potential for enhancing the capacity [1] by using either brand new multicore fibers, defined as space division multiplexing (SDM), or multimode fibers, defined as mode division multiplexing (MDM). The large installed multimode fiber base is seeing an upgrading to newly developed multicore fibers, though, dedicated components such as connector, amplifier, etc. should also be developed correspondingly. Optical MIMO based on coherent detection $[2,3]$ needs local oscillators to interfere with incoming optical fields at the receiver end, in order to keep a linear downconversion process, therefore the complexity and cost are increased considerably, indicating that direct detection is a preferable choice. As far as MDM is concerned, conventional intensity modulation direct detection (IMDD) and subcarrier multiplexing (SCM) schemes show disability as optical fields of different channels spatially overlapped, which would result in detrimental cross terms upon square-law detection. In spite of aforementioned difficulties, the potential capacity brought by multimode fibers is certainly worth the effort.

In this letter, a direct detection implementation using SCM-SS scheme for optical MIMO transmission over multimode fiber links is proposed.

\section{Principle of SCM-SS scheme}

The basic idea of SCM-SS scheme is to overcome intermodal coupling incurred impairment at direct detection regime. Transmitter of each channel consists of subcarrier multiplexing (SCM) and spread spectrum (SS) modulation, denoted as SCM-SS. The motivation of SCM is to produce a modulated single sideband with an optical carrier, see Fig. 1 (a), which can be self-heterodyned through square-law detection, moreover it is able to relieve the impairment of chromatic dispersion. Spread spectrum modulation is to phase modulate the optical field created by SCM with a sequence of spectrum-spreading code, herein each channel is assigned a unique signature.

Assume a MIMO system consisting of $N_{t}$ transmitters and $N_{r}$ receivers. The optical field of each channel at the multimode fiber input side can be expressed as

$$
E_{j}(t)=\exp \left(i \omega_{0} t+i \phi_{j}(t)+i c_{j}(t)\right)\left\{A_{0}+A_{m} \exp \left(i \omega_{m} t+i \varphi_{j}(t)\right)\right\}
$$

where $j=1,2, \ldots, N_{t}$. $\omega_{0}$ is the optical carrier, $\omega_{m}$ is the RF subcarrier, $\phi_{j}(t)$ is the laser phase noise, $c_{j}(t)$ is the spreading code induced phase changing, 


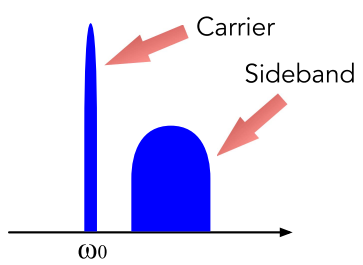

(a)

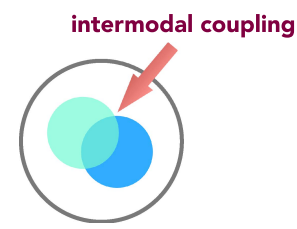

(b)

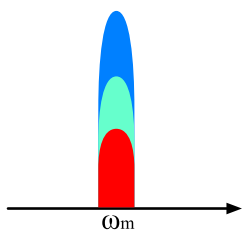

(c)

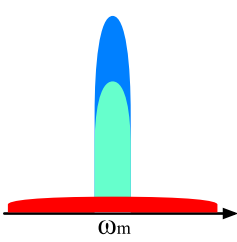

(d)

Fig. 1. Illustration of SCM-SS scheme. $\omega_{0}$ is the optical carrier frequency, $\omega_{m}$ is the RF subcarrier frequency. - Signal from Channel 1. Signal from Channel 2. Impairment. (a) Optical spectrum created by SCM. (b) Two channels coupled together. (c) Electrical spectrum without spread spectrum technique. (d) Electrical spectrum with spread spectrum technique.

$\varphi_{j}(t)$ is the phase shift keyed (PSK) data, $A_{0}$ and $A_{m}$ are amplitude of optical carrier and that of the sideband respectively.

Since different modal power distributions are excited by different channels at the fiber input facet, each channel will experience a different propagation procedure, that is, carried by different set of modes with different fractions of power, until arriving at the receiver side. At the receiver side, every detector receives a collection of optical fields from different channels, attempts of decomposing channels in optical domain are not needed. Optical field impinged on the $i$ th photo detector can be expressed as

$$
\begin{aligned}
U_{i}(t) & =\sum_{j=1}^{N_{t}} E_{j}(t) * g_{i j}=\sum_{j=1}^{N_{t}} E_{j}(t) * \sum_{k=1}^{P} a_{i j k} \delta\left(t-\tau_{k}\right) \\
& =\sum_{j=1}^{N_{t}} \sum_{k=1}^{P} a_{i j k} E_{j}\left(t-\tau_{k}\right)
\end{aligned}
$$

where $i=1,2, \ldots, N_{r}$. * means convolution, $g_{i j}$ is the impulse response associated with $i$ th receiver and $j$ th transmitter, $P$ is the number of modes supported by multimode fiber channel, $a_{i j k}$ is a multiplicative factor, the subscript $i j k$ means from the $j$ th transmitter, via the $k$ th mode, to the $i$ th receiver, and $\tau_{k}$ is the group delay for the $k$ th mode. The square-law-detected photo current, exclusive of dc component and additive noise, is described as

$$
r_{i}(t)=\sum_{j=1}^{N_{t}} A_{i j} \cos \left(\omega_{m} t+\varphi_{j}(t)+\theta_{i j}\right)+\text { s.i. }
$$

where $A_{i j}$ is the resultant amplitude of RF signal from the $j$ th transmitter to the $i$ th receiver, $\theta_{i j}$ is the resultant phase in RF subcarrier, since each channel experienced a different propagation procedure, time delay $\tau_{k}$ was translated into a phase delay $\omega_{m} \tau_{k}, \theta_{i j}$ is apt to be different for each transmitter/receiver pair, in other words, we may take benefit from the multipath scattering environment provided by multimode fiber [2], and s.i. represents 
suppressed impairment. Look back at Eq. (1), the phase noise $\phi_{j}(t)$ in optical carrier of each channel using individual laser behaves independently. Providing optical fields of different channels coupled together, see Fig. 1 (b), upon square-law detection, the interaction of phase noises would also appear in RF band, along with the information-bearing RF signals, see Fig. 1 (c). The impairment from laser phase noises may totally corrupt the transmission. By employing the spread spectrum technique, the phase noise induced impairment is spectrally broadened, so the power of phase noise containing terms in-band are dramatically suppressed, see Fig. 1 (d).

The real-valued bandpass signal in Eq. (3) is turned to complex lowpass equivalent through Hilbert transform. As a result, the sampled (at symbol rate) lowpass equivalent corresponding to the $i$ th receiver can be expressed as a linear combination of transmitted symbols

$$
y_{i}=\sum_{j=1}^{N_{t}} \alpha_{i j} e^{i \theta_{i j}} \cdot x_{j}+n_{i}
$$

where $\alpha_{i j}$ is the sampled amplitude, $x_{j}$ is the symbol from the $j$ th transmitter, $n_{i}$ is the additive noise. It is necessary to mention that in the context of IMDD implementation, such as in $[4,5]$, spatial overlap among fields of different channels should be reduced as much as possible in order to keep a linear relation with respect to optical intensity, otherwise with the advent of cross terms, it is unable to compensate for interferences among channels by MIMO signal processing. SS technique can be incorporated in IMDD to suppress cross terms, though, phase modulated constellations are not supported in the absence of subcarrier. For mathematical convenience, Eq. (4) can be represented in matrix form as

$$
\mathbf{y}=\mathbf{H x}+\mathbf{n}
$$

where $\mathbf{y}=\left[\begin{array}{llll}y_{1} & y_{2} & \ldots & y_{N_{r}}\end{array}\right]^{T}, \mathbf{x}=\left[\begin{array}{llll}x_{1} & x_{2} & \ldots & x_{N_{t}}\end{array}\right]^{T}, \mathbf{H}$ is channel matrix with complex entries of $h_{i j}=\alpha_{i j} e^{i \theta_{i j}}$, and $\mathbf{n}$ accounts for noises. The variation rate of multimode fiber channel is much smaller than the data rate, which is hence categorized into slow fading channel. $\mathbf{H}$ can be steadily tracked by sending training symbols, and is valid for the whole frame duration. All the channels are frame synchronized, and each channel is allocated with a unique sequence of training symbols. Transmitted symbols are recovered by zero forcing algorithm

$$
\hat{\mathbf{x}}=\left(\mathbf{H}^{H} \mathbf{H}\right)^{-1} \mathbf{H}^{H} \mathbf{y}
$$

where $\hat{\mathbf{x}}$ is an estimation of $\mathbf{x}, \mathbf{H}^{H}$ is the conjugate transpose of $\mathbf{H}$.

\section{Experimental implementation}

The feasibility of this approach is verified experimentally by a $2 \times 4 \mathrm{MIMO}$ system with moderate data rate, so as to alleviate other degradations. On the other hand, from a statistical viewpoint, $2 \times 4$ MIMO can achieve higher diversity gain than $2 \times 2 \mathrm{MIMO}$, which is thought to improve communication 


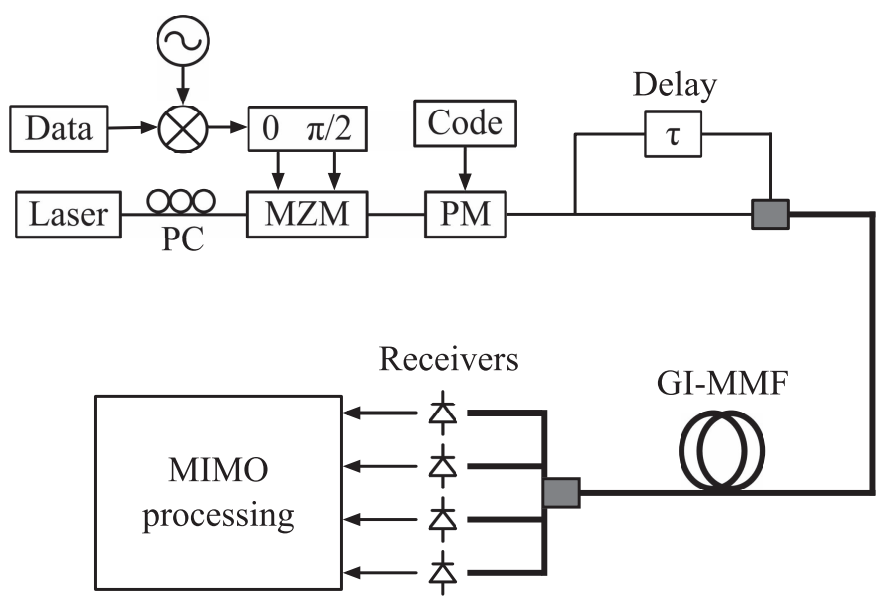

Fig. 2. Experimental setup. PC: polarization controller, MZM: Mach-Zehnder modulator, PM: phase modulator.

reliability, as also observed in [6]. The experimental setup is shown in Fig. 2, multimode fiber parts are indicated by bold lines.

A $1550 \mathrm{~nm}$ DFB laser acts as the light source. $100 \mathrm{Mbps}$ binary phase shift keyed (BPSK) data is moved to passband by $2 \mathrm{GHz}$ RF subcarrier, the $\mathrm{RF}$ signal is then split to two orthogonal arms by a $\pi / 2$ hybrid coupler. A dual-drive MZM is biased at quadrature point, and the two arms of MZM are modulated by the two orthogonal electrical signals respectively. Suppression of the unwanted sideband for more than $15 \mathrm{~dB}$ was achieved. This optical field is then phase modulated by a sequence of spectrum-spreading code with 10 Gcps. The optical signal is split into two channels, one of the channels is delayed by $2 \mathrm{~km}$ single mode fiber with the intention of decorrelating the two channels. The two channels carried by single mode fibers are coupled to the input ports of a fused multimode coupler through connectors, which will excite lower order modes of $50 \mu \mathrm{m}$ core diameter graded index multimode fiber (GI-MMF) link. Different link lengths are constituted by $500 \mathrm{~m}$ and $1000 \mathrm{~m}$ fiber reels, which are connected by optical connectors. The output optical fields are directed to 4 photo detectors by another fused multimode coupler. Each detector receives a mixing of signals from both channels. The electrical signals are sampled with $20 \mathrm{GHz}$ for off-line MIMO signal processing. In experiments 16 symbols of $2^{7}-1$ pseudo-random binary sequence (PRBS) are taken as training symbols, and $2^{7}-1$ symbols constitute one frame for each channel.

\section{Experimental results}

Up to $2000 \mathrm{~m}$ transmissions have been achieved via SCM-SS scheme. To confirm the advantages of the proposed SCM-SS scheme further, the experiments of SCM scheme that using no spread spectrum technique are also conducted, and corresponding received and recovered constellations of $2000 \mathrm{~m}$ transmission under both schemes are shown exemplarily in Fig. 3 (a).

Notice that the points of the SCM-SS scheme cluster closely, indicating 


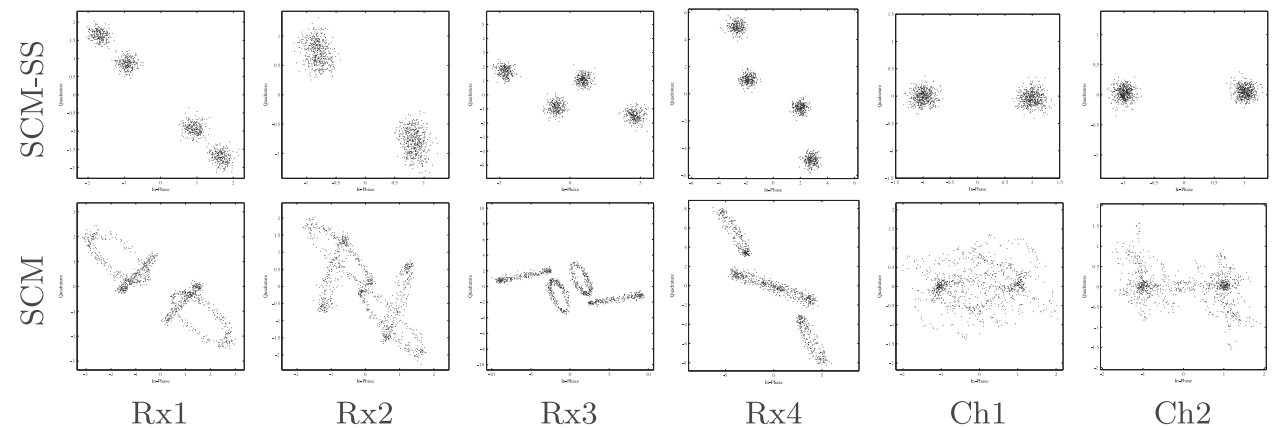

(a) Constellation diagrams of $2000 \mathrm{~m}$ transmission. Rx: receiver, Ch: channel

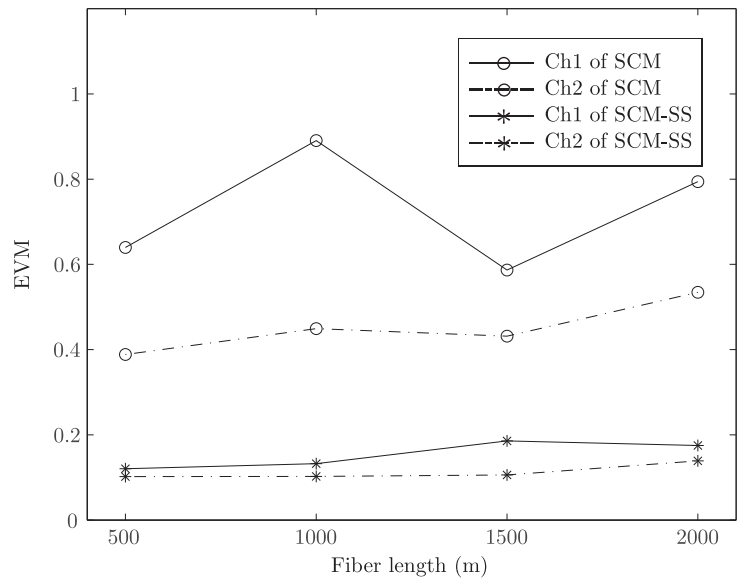

(b) EVM of different link lengths.

Fig. 3. Experimental results.

good quality of signals, meanwhile different styles of received constellations in SCM-SS scheme manifest modal-coupling diversity present at the output end of multimode fiber. The condition number of the channel matrix $\kappa(\mathbf{H})$ in this example is 3.5. Whereas the trajectories on the constellations of SCM scheme clearly reveal the influence of phase noises.

Fig. 3 (b) illustrates the error vector magnitude (EVM) of both schemes under different link lengths. Of which $1500 \mathrm{~m}$ and $2000 \mathrm{~m}$ transmissions are comprised of $1000+500 \mathrm{~m}$ and $1000+500+500 \mathrm{~m}$ fibers respectively. Error free demodulation of SCM-SS scheme was verified, and superior performance compared with SCM scheme is apparently exhibited through much lower EVM. When switching to different link lengths, fiber reels and connectors should be reorganized accordingly, though, stringent adjustments were not exercised, manifesting the tolerances to channel fluctuations to some extent. To evaluate the performance further, the statistics of multimode fiber channel should also be taken into account [7], which is a topic of future investigation.

\section{Conclusions}

Satisfactory performance of $2 \times 4$ optical MIMO using SCM-SS scheme was demonstrated in the presence of intermodal coupling, showing its applicability of capacity enhancement in multimode fiber links. 